\title{
Ion-Specific Clustering of Metal-Amphiphile Complexes in Rare Earth Separations
}

\author{
Srikanth Nayak, Kaitlin Lovering, and Ahmet Uysal * \\ Chemical Sciences and Engineering Division, Argonne National Laboratory, Argonne IL 60439 \\ *Email: ahmet@anl.gov. Web: www.anl.gov/profile/ahmet-uysal

\section{KEYWORDS} \\ Solvent extraction, small angle X-ray scattering, specific ion effects, complex fluids, amphiphile \\ aggregation
}




\section{ABSTRACT}

Aggregation and clustering of metal-amphiphile complexes formed during solvent extraction of lanthanides have been studied with small angle X-ray scattering. The nanoscale structure of the complex fluid strongly depends on the counter-ion $\left(\mathrm{NO}_{3}{ }^{-}\right.$or $\left.\mathrm{SCN}^{-}\right)$and the lanthanide being extracted. As a result, it is possible to selectively transport light or heavy lanthanides from the aqueous phase into the organic phase by simply choosing $\mathrm{NO}_{3}^{-}$or $\mathrm{SCN}^{-}$as the background anion, respectively. While the organic phase containing TOMA-NO 3 always shows clustering, indicating the presence of stronger attractive interactions between metal-amphiphile aggregates, TOMASCN shows clustering as a function of the metal loading. These qualitative differences suggest that the extraction efficiency is driven by the aqueous phase conditions in $\mathrm{NO}_{3}{ }^{-}$solutions, while it is driven by the organic phase structuring in $\mathrm{SCN}^{-}$solutions. A clustering model, that accounts for the hard sphere repulsions and short-range attractions between the aggregates, has been developed to model the X-ray scattering results. The new model successfully describes the nanoscale structure and helps understanding the mechanisms responsible for amphiphile assisted ion transport and complexation between immiscible liquids. 
The demand for lanthanide elements is rising due to their use in many technological applications, such as permanent magnets used in electric vehicles and wind turbines. ${ }^{1}$ This has provided an impetus to research into efficient separation of lanthanides from their mixtures. ${ }^{2-4}$ Industrially, solvent extraction is the most common separation technology used in refining and reprocessing of lanthanides and other heavy elements. ${ }^{5-7}$ Solvent extraction (SX) works on the principle of preferential distribution of a solute between two immiscible liquids (Figure 1a). The metal ions are transferred from an aqueous phase into an organic phase with the help of extractant molecules, which are typically amphiphilic molecules that form reverse micellar aggregates (referred to as aggregates) with the transferred metal ions, co-transported anions, and water molecules (Figure 1b). The complex fluid formed by these aggregates play a crucial role in the overall efficiency of the SX process, yet its molecular scale structure is not well-understood. ${ }^{8}$ Here, SAXS experiments reveal that the character of the anions has huge effects on the interactions between the aggregates and their clustering behavior in the complex fluid, which eventually determines heavier or lighter lanthanides are extracted preferentially.

Lanthanide ions are typically trivalent and have very similar chemical properties, which make their separation from each other very challenging. Nevertheless, choice of extractant and the ionic conditions in the aqueous medium, such as the character of the background anion, can exploit the subtle differences between lanthanides, such as ionic radius and hydration enthalpy, for preferential extraction of one lanthanide over another. With acidic extractants, which bind to the metal in an inner sphere coordination manner, extraction generally increases with atomic number (Figure 1c). ${ }^{9-11}$ This is commensurate with the increasing electrostatic interaction with decreasing ionic radius of the lanthanide as interactions between lanthanides and anions are predominantly electrostatic. ${ }^{12}$ With neutral and basic extractants, where the extractant binds to a neutral or anionic 
complex of the lanthanide, the anions can influence selectivity dramatically (Figure 1c). Several hypotheses have been put forward to explain these trends. A balance between the electrostatic interactions and the steric repulsions between the anions has been suggested to explain the bellshape in extraction trends with neutral extractants (Figure 1c, dataset C), where middle lanthanides are extracted better than light or heavy lanthanides. ${ }^{13}$ Recent results with N,N,N,N-tetra(noctyl)diglycolamide, a neutral extractant, show that the anions bind to the metal in an outer sphere manner with extractant in the inner sphere. ${ }^{14-15}$ These results highlight the fact that structure of complexes formed in extraction and the role of anions can be more complicated than those expected by simple electrostatic arguments.
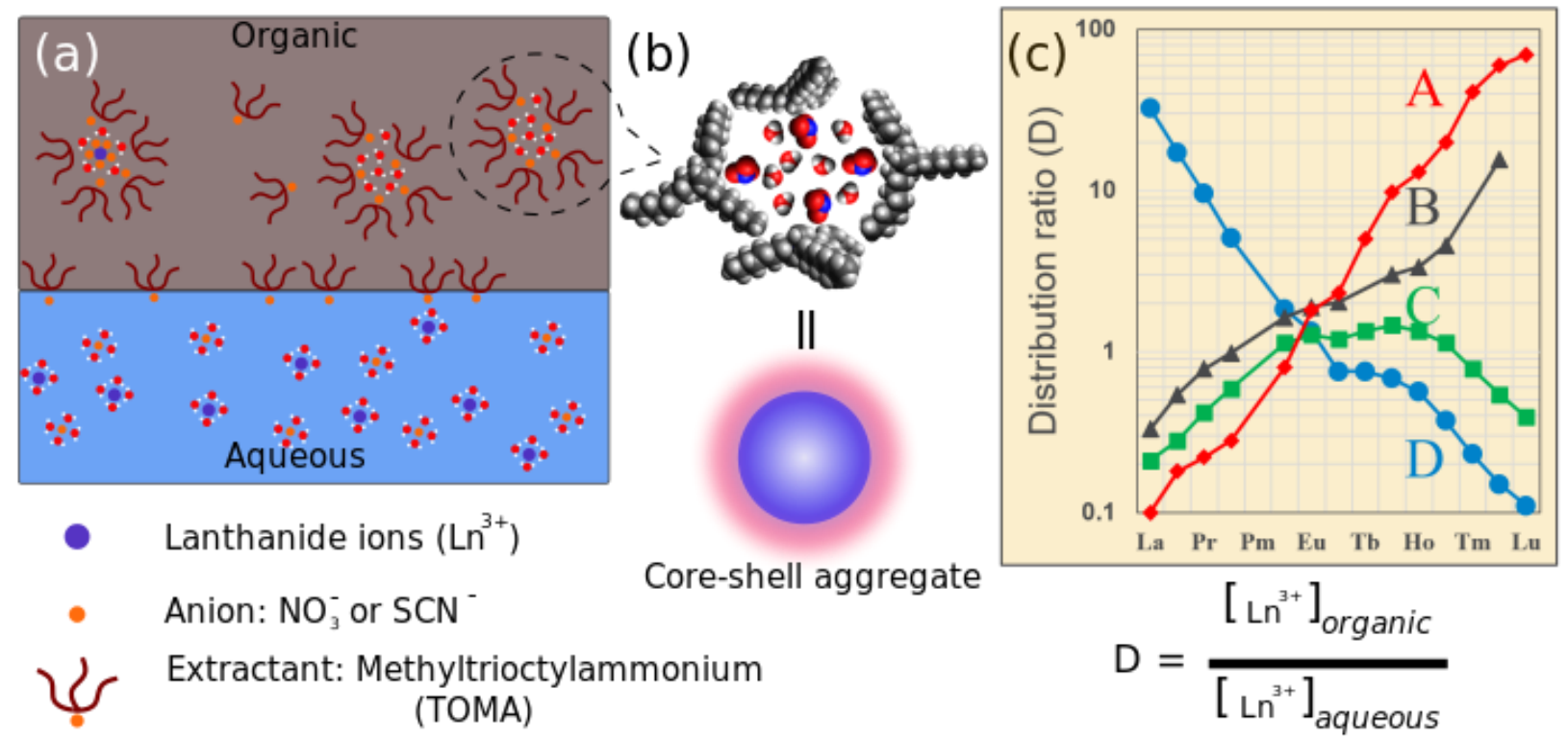

Figure 1 (a) Schematic depicting solvent extraction process. Amphiphilic extractants facilitate the transfer of metal ions from the aqueous to the organic phase. (b) Extractants aggregate in the organic phase to form core-shell structures with extractant headgroups, water and ionic species in the core, and extractant tails forming the shell. (c) Extraction efficiency of lanthanides across the series with different extractants: A - di-(2-ethylhexyl)phosphoric acid (D2EHPA) extracting from $\mathrm{HNO}_{3}$ medium (adapted from Sato, Hydrometallurgy, 22, 1989 ${ }^{11}$ ), B - Aliquat 336. SCN 
extracting from KNCS medium, $\mathrm{C}$ - tributylphosphate (TBP) extracting from nitrate medium, and D - Aliquat 336. $\mathrm{NO}_{3}$ extracting from $\mathrm{NaNO}_{3}$ medium. Data for $\mathrm{B}, \mathrm{C}$ and $\mathrm{D}$ are obtained from $\mathrm{Du}$ Preez and Preston, Solvent Extraction, $1990 .{ }^{13}$ Acidic extractants, such as D2EHPA, typically show increasing extraction trend across the series. Neutral extractants like TBP show aniondependent extraction behavior. Anion-dependence is striking in Aliquat 336 where $\mathrm{NO}_{3}{ }^{-}$and $\mathrm{SCN}^{-}$ show reverse extraction trends.

With respect to the effect of anions on SX, basic extractants show a significantly different behavior compared to the acidic or neutral extractants (Figure 1c, datasets B and D). ${ }^{13,16-17}$ Solvent extraction of lanthanides with Aliquat 336 (a quaternary ammonium) shows decreasing distribution ratio with atomic number when extracted from a $\mathrm{NO}_{3}{ }^{-}$medium, and the reverse when extracted from a $\mathrm{SCN}^{-}$medium. ${ }^{13}$ The decreasing trend with $\mathrm{NO}_{3}{ }^{-}$background is unique and cannot be explained by either electrostatic effects or steric arguments. ${ }^{18}$ Aggregation of extractants has been hypothesized to influence the extraction trends and anion effects in the case of Aliquat 336. ${ }^{19}$ The salting-out effect in the aqueous phase also plays a role in determining the organic phase speciation and extraction efficiency. ${ }^{18-21}$ Further, it has been suggested that in both $\mathrm{SCN}^{-}$ and $\mathrm{NO}_{3}{ }^{-}$cases, aggregates of Aliquat 336 extract the lanthanide rather than single extractant molecules. ${ }^{19}$ However, the aggregation structure in these systems has not been characterized. Aggregation of quaternary ammonium surfactants used in nanoparticle synthesis is known to affect the morphology of formed nanoparticles. ${ }^{22-23}$

Characterization of the nanostructure of organic phase after solvent extraction, a complex fluid with high concentration of polar solutes such as ions and water, amphiphilic extractants and nonpolar diluents, is non-trivial. Small angle X-ray and neutron scattering (SAXS and SANS, respectively) have been the principal techniques in this effort and they show the link between 
aggregation structures on extraction efficiency and third phase formation (an undesired fluid-fluid phase transition and splitting due to high metal loading) in different extraction systems. ${ }^{24-42} \mathrm{~A}$ significant portion of these studies used Baxter's sticky hard sphere model to describe the interactions between reverse micellar (RM) aggregates. In Baxter model, spherical aggregates attract each other when they are very close and show hard sphere repulsion when the distance between their centers is less than or equal to their hard sphere diameter (See results and discussion for details). Although Baxter model has been successful in capturing certain trends and relations between the molecular scale structure and macroscopic observables, it has been criticized for overestimating the interactions between the aggregates. ${ }^{43-44}$ According to Baxter model, when aggregates form clusters, they should have long range ordering which should lead to peaks in the high-q region of the SAXS data. However, this is almost never observed in SX systems, even when the increasing slope in the low-q region of the SAXS data suggested clustering. One simple reason for this discrepancy may be that the extractant-metal aggregates are not hard spheres and their shape may deform while they are clustering.

In this study, we report SAXS studies of variations in the structure of organic phase containing quaternary ammonium extractants (TOMA, Figure 1c, data sets B and D), with varying lanthanide and background anion conditions. A modified model based on the clustering of interacting hard spheres, which eliminates the drawbacks of the Baxter model, is used to interpret the trends in the SAXS data. Our results show that the structure of extractant-metal aggregates varies dramatically based on the character of the anion being $\mathrm{NO}_{3}{ }^{-}$or $\mathrm{SCN}^{-}$. Even without any lanthanide extracted, the TOMA-NO 3 shows clustering of the aggregates driven by attractive interactions between them. The TOMA-SCN system shows a predominantly hard sphere interaction between the aggregates indicating strong repulsion between them. The preference of light (heavy) lanthanides in $\mathrm{NO}_{3}$ 
$\left(\mathrm{SCN}^{-}\right)$containing systems during $\mathrm{SX}$ appears to be a direct result of these differences. The clustering behavior of TOMA-NO 3 does not change with lanthanide extraction, indicating that the attraction between the aggregates is not affected. In contrast, increasing metal extraction increases the clustering of the aggregates in the TOMA-SCN. These results suggest that there are multiple factors that account for the different lanthanide extraction behaviors with $\mathrm{NO}_{3}{ }^{-}$and $\mathrm{SCN}^{-}$, and lanthanides possibly follow different pathways in the free energy landscape during the transfer between the aqueous and the organic phases based on the character of the anion.

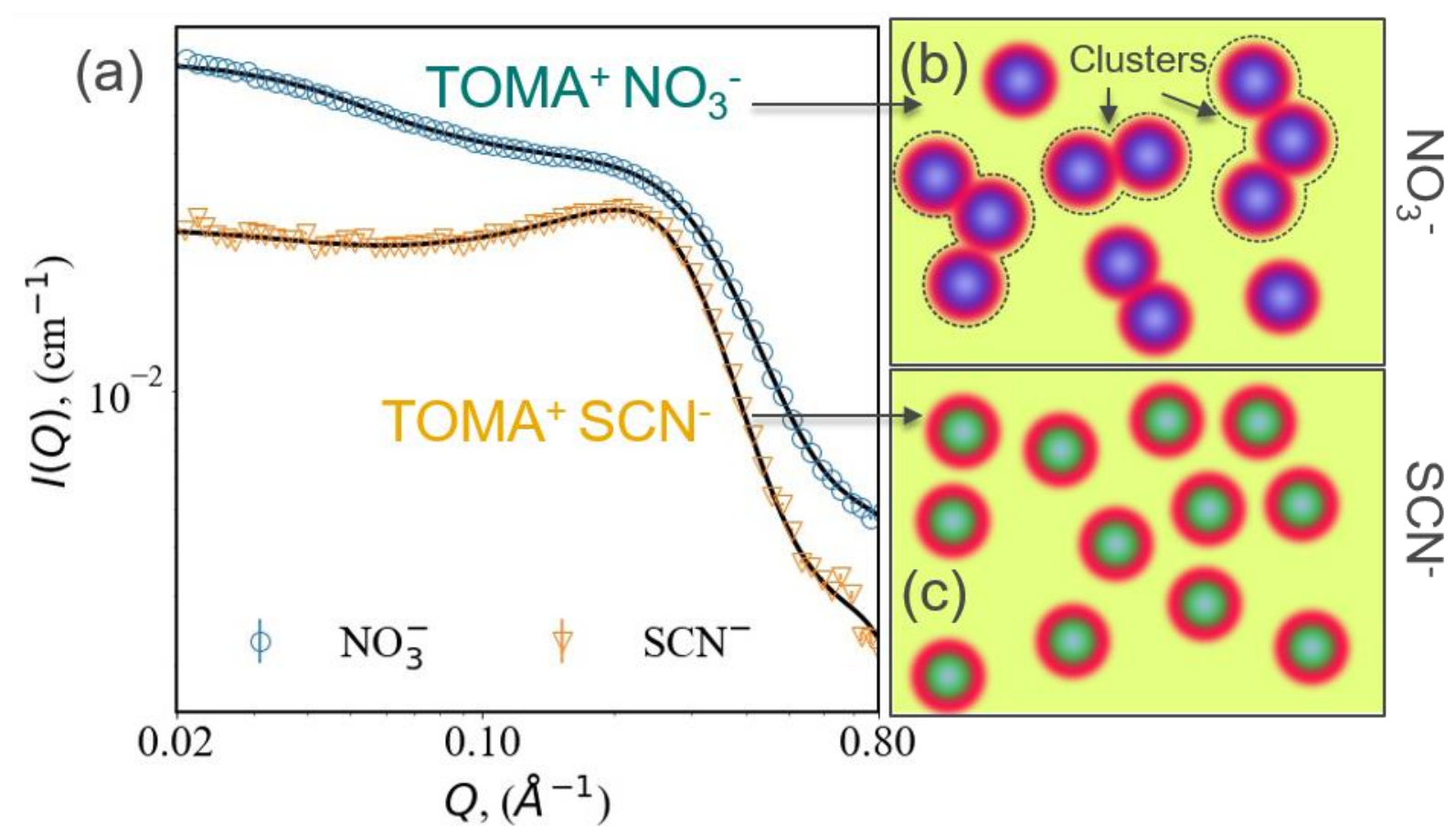

Figure 2. (a) SAXS profiles of $0.25 \mathrm{M}$ TOMA-NO 3 (blue circles) and TOMA-SCN (yellow triangles) dissolved in toluene and contacted with $3 \mathrm{M} \mathrm{NaNO}_{3}$ and $3 \mathrm{M} \mathrm{NaSCN}$ aqueous solutions respectively. The solid black lines show the fit obtained from the hard sphere clustering model. The fit parameters are tabulated in Table. S1. (b) Schematic representing the clustering of TOMA-NO 3 aggregates in toluene, and (c) schematic representing the hard sphere fluid structure of TOMA-SCN aggregates in toluene. 


\section{RESULTS AND DISCUSSIONS}

First, we investigate the aggregation and clustering of $0.25 \mathrm{M}$ TOMA in toluene after contacting it with $3 \mathrm{M} \mathrm{NaNO}_{3}$ and $3 \mathrm{M} \mathrm{NaSCN}$ aqueous solutions, without any lanthanide ions (Figure 2a). Even without any modeling, the qualitative differences between the data sets are clear. A detailed analysis requires an overall discussion of SAXS data modelling.

SAXS data for the organic phases obtained from SX has been traditionally analyzed with models of interacting particles. As the metal concentration in the organic phase increases, a combination of the following can occur: 1) aggregates can undergo shape changes due to the changed polarity of the cores, or 2) aggregates retain their shape, but the interactions between the aggregates change leading to a mesoscale change in the complex fluid structure. Since both the phenomena lead to similar changes in scattering behavior, namely, increased low-Q scattering, decoupling them is inherently challenging in SAXS analysis. Thus, some studies have analyzed the SAXS data on the basis of aggregate shape change using inverse Fourier transform techniques, ${ }^{32,45-46}$ while others have fixed the aggregate shape and analyzed the changes in inter-micellar interactions using the sticky hard spheres model. The Baxter model for hard spheres with short-range attractive interactions has been extensively used due to its prediction of phase changes, analogous to the third phase formation in SX. ${ }^{25-26,47}$ Despite its successes, the Baxter model has been challenged recently. ${ }^{43-44}$ In fact, Baxter model is known to overestimate the strength of attraction between the aggregates. At low values of the stickiness parameter (strong attraction), the Baxter model leads to stronger hard sphere peaks due to the increased proximity of hard spheres. However, the assumption that aggregates remain as hard spheres, even when strongly attracting each other, may not be valid. We found Baxter model to be inadequate in describing the structural changes in the TOMA-NO 3 systems (Figure S3). In addition to its failure to qualitatively capture the SAXS data, 
Baxter model, generally, requires unrealistically low hard sphere volume fractions to be able to fit SAXS data. To overcome these shortcomings, trajectories from molecular dynamics simulations have been used to interpret the SAXS results. ${ }^{36,}{ }^{44}$ However, no analytical models have been provided as an alternative to the Baxter model.

We use a model based on clustering of hard spheres driven by an anonymous attractive interaction. As the attraction between colloidal hard spheres strengthens, they start to form clusters. These clusters lead to an increased scattering at low-Q values in SAXS profiles, whose exact form depends on the cluster morphology. The model superimposes a cluster shape function (corresponding to attraction between aggregates) on a hard sphere fluid structure (corresponding to repulsion between the aggregates). ${ }^{48}$ Various theoretical profiles such as Lorentzian and Guinier-Porod model have been used to analyze the clustering behavior of attractive colloids. ${ }^{48-49}$ We use the Guinier-Porod model to describe the shape of clusters. Details of the model is explained in the methods section.

We apply this model to the data in Figure 2a (solid lines). The different scattering pattern for $\mathrm{NO}_{3}{ }^{-}$and $\mathrm{SCN}^{-}$systems indicate that the organic phase is structured in both the cases and the structure depends on the character of the anion. While TOMA-SCN appears to have a clear hard sphere peak, TOMA- $\mathrm{NO}_{3}$ has a subdued hard sphere peak and an increased scattering in the lowQ region. The size of the core $\left(\mathrm{R}_{\mathrm{c}} \sim 3 \AA\right)$ and the shell $\left(\mathrm{R}_{\mathrm{s}} \sim 10 \AA\right)$ of the aggregates are similar for TOMA-NO 3 and TOMA-SCN (Table S1). A recent study based on slope analysis suggested that aggregation numbers are lower in TOMA- $\mathrm{NO}_{3}$ compared to TOMA-SCN. ${ }^{19}$ However, slope analysis may not be very accurate in non-ideal conditions. ${ }^{50}$

The main difference between $\mathrm{NO}_{3}{ }^{-}$and $\mathrm{SCN}^{-}$is in the apparent hard sphere volume fraction $\left(\phi_{\mathrm{HS}}\right)$ and the number of particles per cluster $(\mathrm{f}+1)$. While $\phi_{\mathrm{HS}}$ in TOMA-SCN $(0.129)$ is close to the 
volume fraction of extractant $(\sim 0.13)$, it is much lower for TOMA-NO $\mathrm{NO}_{3}(0.068)$. Further, TOMA$\mathrm{NO}_{3}$ shows a higher clustering behavior than TOMA-SCN as indicated by the corresponding increase in low-Q scattering. Average number of aggregates per cluster is 1.7 and 1 for TOMA$\mathrm{NO}_{3}$ and TOMA-SCN, respectively. In a hard sphere fluid, the height of the hard sphere peak corresponds to the volume fraction of hard spheres. Since the extractant concentration (volume fraction) is the same in TOMA-NO $\mathrm{NO}_{3}$ and TOMA-SCN, the lower $\phi_{\mathrm{HS}}$ in TOMA-NO $\mathrm{T}_{3}$ system (higher clustering) suggests that hard sphere repulsions are weaker. The assumption that the aggregates behave as hard spheres when they are in close contact may not be valid. Another way to understand the decrease in $\phi_{\mathrm{HS}}$ is to consider the presence of clusters in a hard sphere fluid as an effective increase in polydispersity in the system. It has been shown that increasing polydispersity in hard sphere fluids leads to a decrease in the peak height corresponding to the nearest neighbor interactions. ${ }^{51-52}$ Porod exponent of $\sim 1$ indicates that the clusters are elongated or cylindrical. Clustering of micelles and aggregates to form elongated structures in response to changes in medium conditions have been reported, ${ }^{53-55}$ and have been proposed as an alternative to the globular clustering predicted by the Baxter model in malonamide, ${ }^{32,34,45}$ and in TBP extraction systems. ${ }^{56}$

Aggregation of amphiphiles in nonpolar media such as toluene is favored by interactions between the polar head groups and disfavored by the loss of entropy upon aggregation. ${ }^{57}$ Hydrogen bonding between the head groups, sometimes mediated by water, also stabilizes the aggregates. ${ }^{44}$ 46 The differences in head group interactions, hydration, and hydrogen bonding environments between TOMA-NO 3 and TOMA-SCN are the likely causes of their different aggregation structures. For instance, we have recently shown that at the air/aqueous interface, $\mathrm{NO}_{3}{ }^{-}$and $\mathrm{SCN}^{-}$ ions show qualitatively different adsorption behavior at quaternary amine Langmuir monolayers. 
A combined X-ray scattering and vibrational sum frequency generation (VSFG) study showed that $\mathrm{NO}_{3}{ }^{-}$ions can adsorb without disturbing the hydrogen bonding network at the interface, while $\mathrm{SCN}^{-}$causes an apparent dehydration of the interface. It is reasonable to assume that oxygen in $\mathrm{NO}_{3}{ }^{-}$are more compatible with the hydrogen bonding network of water molecules. The lack of similar compatibility probably leads to a more rigid core in TOMA-SCN aggregates, preventing clustering. Nevertheless, extended X-ray absorption fine structure (EXAFS) experiments in future studies will be helpful in obtaining a clear picture of the nanostructure within these aggregates and understanding the cause of these different clustering behaviors with $\mathrm{NO}_{3}{ }^{-}$and $\mathrm{SCN}^{-}$. 

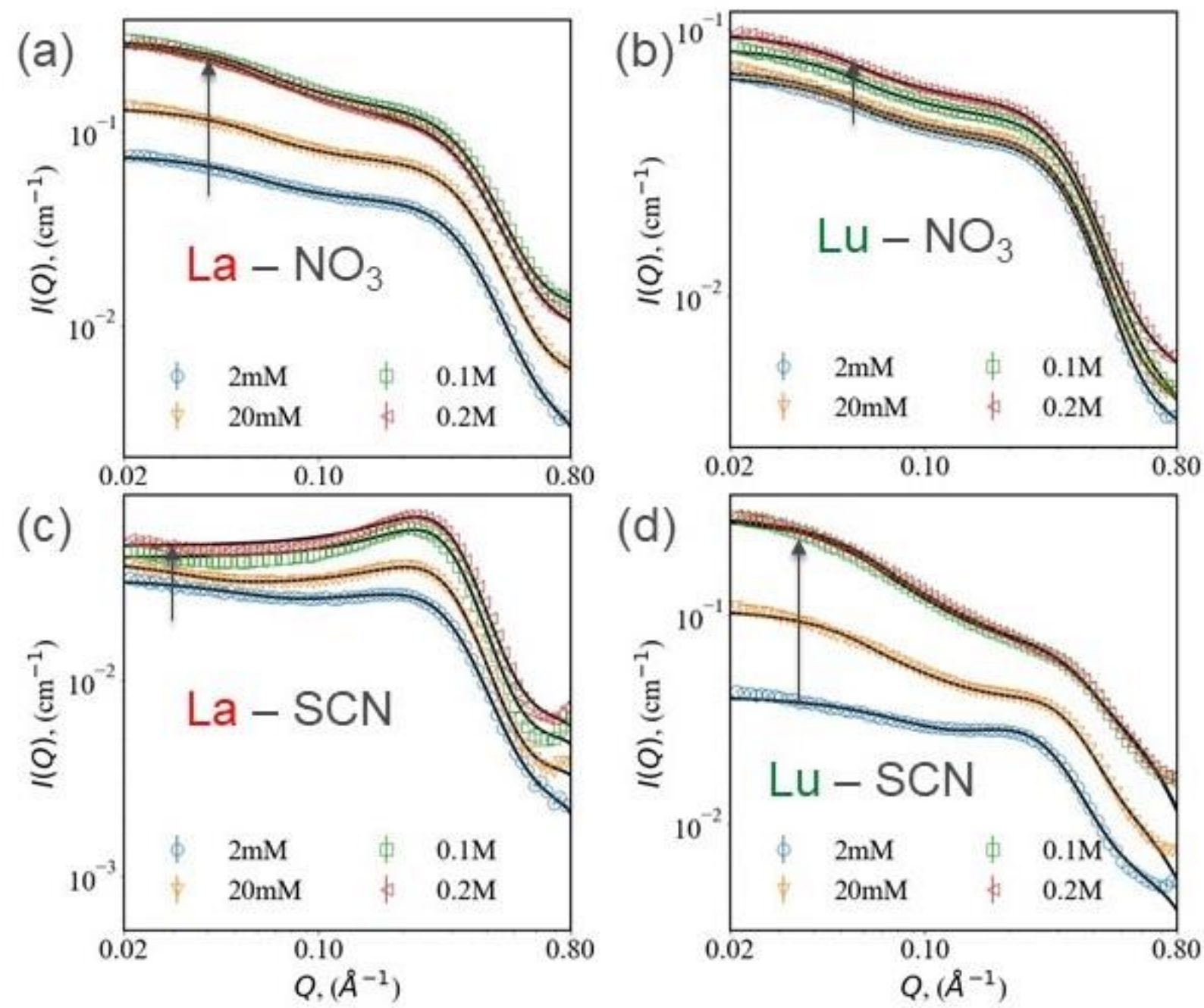

Figure 3. SAXS results for the organic phase obtained after extracting varying (aqueous phase) concentrations of (a) $\mathrm{La}^{3+}$ from $\mathrm{NO}_{3}{ }^{-}$medium, (b) $\mathrm{Lu}^{3+}$ from $\mathrm{NO}_{3}{ }^{-}$medium, (c) ) $\mathrm{La}^{3+}$ from $\mathrm{SCN}^{-}$ medium, and (d) $\mathrm{Lu}^{3+}$ from $\mathrm{SCN}^{-}$medium. There are no significant qualitative differences in SAXS for the $\mathrm{NO}_{3}{ }^{-}$case, irrespective of the changes in extraction behavior. In the $\mathrm{SCN}^{-}$case however, $\mathrm{La}^{3+}$ and $\mathrm{Lu}^{3+}$ show significantly different scattering profiles. While $\mathrm{La}^{3+}$ system shows mainly hard sphere structure, $\mathrm{Lu}^{3+}$ shows increasing clustering with increasing extraction. Arrows in each subplots show the direction of increasing $\left[\mathrm{Ln}^{3+}\right]_{\mathrm{aq}}$. 
Next, we investigate the metal loading dependent evolution of aggregates, using $\mathrm{La}^{3+}$ and $\mathrm{Lu}^{3+}$ as the representative of light and heavy lanthanides, respectively. We observe a strong dependence on the counter-ion present with TOMA. Figure 3 shows the effect of increasing concentration of lanthanides in the aqueous phase $\left(\left[\mathrm{Ln}^{3+}\right]_{\mathrm{aq}}\right)$ on SAXS of the organic phase obtained after SX. In all the cases, increasing $\left[\mathrm{Ln}^{3+}\right]_{\text {aq }}$ leads to an increase in total scattering, due to the higher $\left[\operatorname{Ln}^{3+}\right]_{\text {org }}$ after extraction. When extracting from a $\mathrm{NO}_{3}{ }^{-}$medium, there is no qualitative difference in the scattering profiles of samples without any lanthanides, and those with increasing concentrations of $\mathrm{La}^{3+}$ or $\mathrm{Lu}^{3+}$ in the organic phase (Figure 3a, and b). Samples with $\mathrm{La}^{3+}$ show higher increase in absolute scattering with increasing aqueous phase concentration than those with $\mathrm{Lu}^{3+}$. This is commensurate with the higher extraction of $\mathrm{La}^{3+}$ from nitrate medium compared to $\mathrm{Lu}^{3+}$ (Figure 1

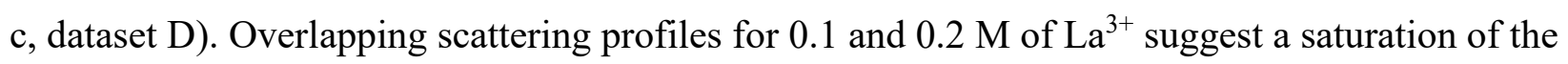
organic phase with $\sim 0.1 \mathrm{M}_{\text {of }} \mathrm{La}^{3+}$ in the $\mathrm{NO}_{3}{ }^{-}$case. In the $\mathrm{SCN}^{-}$case different scattering profiles are seen for $\mathrm{La}^{3+}$ and $\mathrm{Lu}^{3+}$ extraction (Figure 3c, and d). There is a slight reduction in the low-Q scattering behavior at high $\mathrm{La}^{3+}$ concentrations. This change could be due to the poor extraction of $\mathrm{La}^{3+}$ from the $\mathrm{SCN}^{-}$medium, and the increased ionic strength of the aqueous phase at higher $\left[\mathrm{Ln}^{3+}\right]_{\mathrm{aq}}$. Increasing $\left[\mathrm{Lu}^{3+}\right]_{\mathrm{aq}}$ leads to increased low-Q scattering, indicating increasing clustering

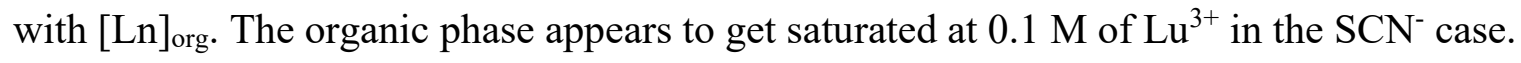

Aggregates are formed with TOMA even in the absence of lanthanides. Hence, it is likely that the lanthanide loaded organic phases contain at least two types of aggregates- with or without the metalate complex. For simplicity, we assume that the all the aggregates are identical in terms of their morphology and inter-aggregate interactions. Thus, the fit parameters should be read as weighted averages of these two populations. Parameters obtained from fitting the clustering model to the data shown in Figure 3 are tabulated in Table S1. In the $\mathrm{NO}_{3}{ }^{-}$case, there is no change in the 
size of the either the core or the shell of the aggregate, but there is an increase in the electron density of the core with increasing $[\mathrm{Ln}]_{\mathrm{aq}}$. There is also a slight increase in the number of aggregates per cluster with increasing $\left[\mathrm{La}^{3+}\right]_{\text {aq. }}$. Similarly, there is no evidence for aggregate shape changes with increasing $\left[\mathrm{Ln}^{3+}\right]_{\mathrm{aq}}$ in the $\mathrm{SCN}^{-}$case as well. With increasing $\left[\mathrm{Lu}^{3+}\right]_{\mathrm{aq}}$ there is an increase in the number of aggregates per cluster, unlike with $\left[\mathrm{La}^{3+}\right]_{\mathrm{aq}}$.
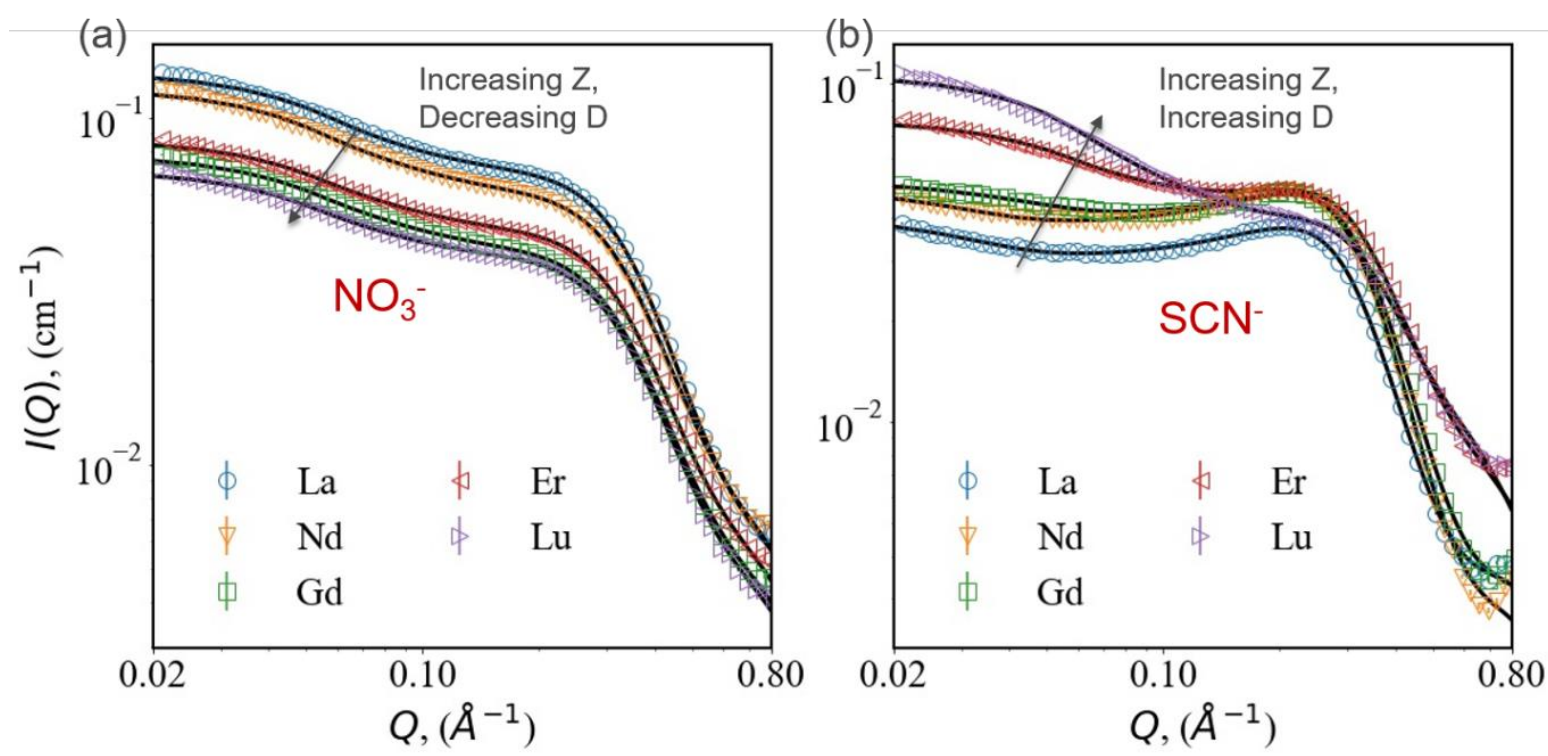

Figure 4. SAXS curves for the organic phase obtained after solvent extraction of $0.02 \mathrm{M}$ of $\mathrm{Ln}^{3+}$ from $3 \mathrm{M}$ (a) $\mathrm{NO}_{3}{ }^{-}$, and (b) $\mathrm{SCN}^{-}$background solutions. The arrows indicate the direction of increasing atomic number.

We studied the variation in organic phase structure with extraction of 5 lanthanides, as representatives of the whole series, from $\mathrm{NO}_{3}{ }^{-}$and $\mathrm{SCN}^{-}$media. Aqueous phase lanthanide concentration was kept at $0.02 \mathrm{M}$. The SAXS data of the corresponding organic phases after SX are shown in Figure 4. With $\mathrm{NO}_{3}{ }^{-}$background, there are no major differences in scattering profiles between the lanthanides (Figure 4a). In the $\mathrm{SCN}^{-}$case, however, there is an increase in clustering, as evidenced by an increase in low-Q scattering, with Er and Lu following the order of extraction 
selectivity (Figure 4b). Further, the hard sphere peak appears to diminish concomitantly. At the highest $[\mathrm{Ln}]_{\text {org }}$ obtained with $\mathrm{Lu}^{3+}$ in $\mathrm{SCN}^{-}$case, the scattering profile is similar to that with $\mathrm{NO}_{3}$.
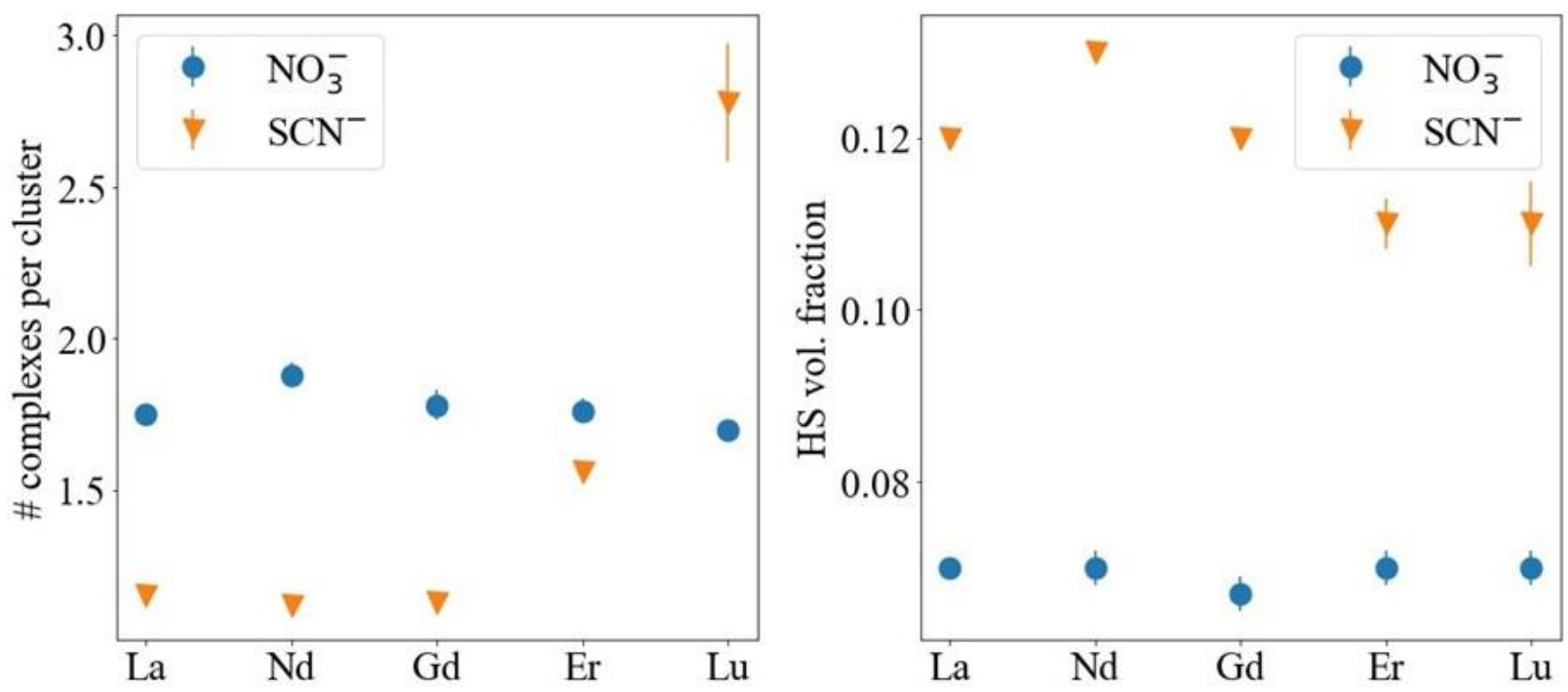

Figure 5. Parameters obtained from fitting the SAXS curves with clustering model. Samples are organic phases obtained by solvent extraction of $0.2 \mathrm{M}$ lanthanides from NO3- (circles), and SCN(triangles) background. (a) Number of aggregates per cluster $=\mathrm{f}+1$, and (b) apparent hard sphere volume fraction $\left(\phi_{H S}\right)$.

The trends observed in the SAXS data can be visualized by plotting the important fit parameters from Table S1. Variation of the clustering parameter $(f+1)$ and the apparent hard sphere volume fraction $\left(\phi_{H S}\right)$ obtained from the fits is shown in Figure 5. These results show that there are qualitative differences between the structure of organic phases containing $\mathrm{NO}_{3}{ }^{-}$and $\mathrm{SCN}^{-}$anions. The clustering behavior appears to follow the extraction trends in the $\mathrm{SCN}^{-}$case, but not in the case of $\mathrm{NO}_{3}^{-}$(Figure 5a). Similarly, $\phi_{H S}$ shows a decreasing trend in $\mathrm{SCN}^{-}$case but remains at an almost constant value of $\sim 0.066$ in the case of $\mathrm{NO}_{3}{ }^{-}$background (Figure $5 b$ ). 

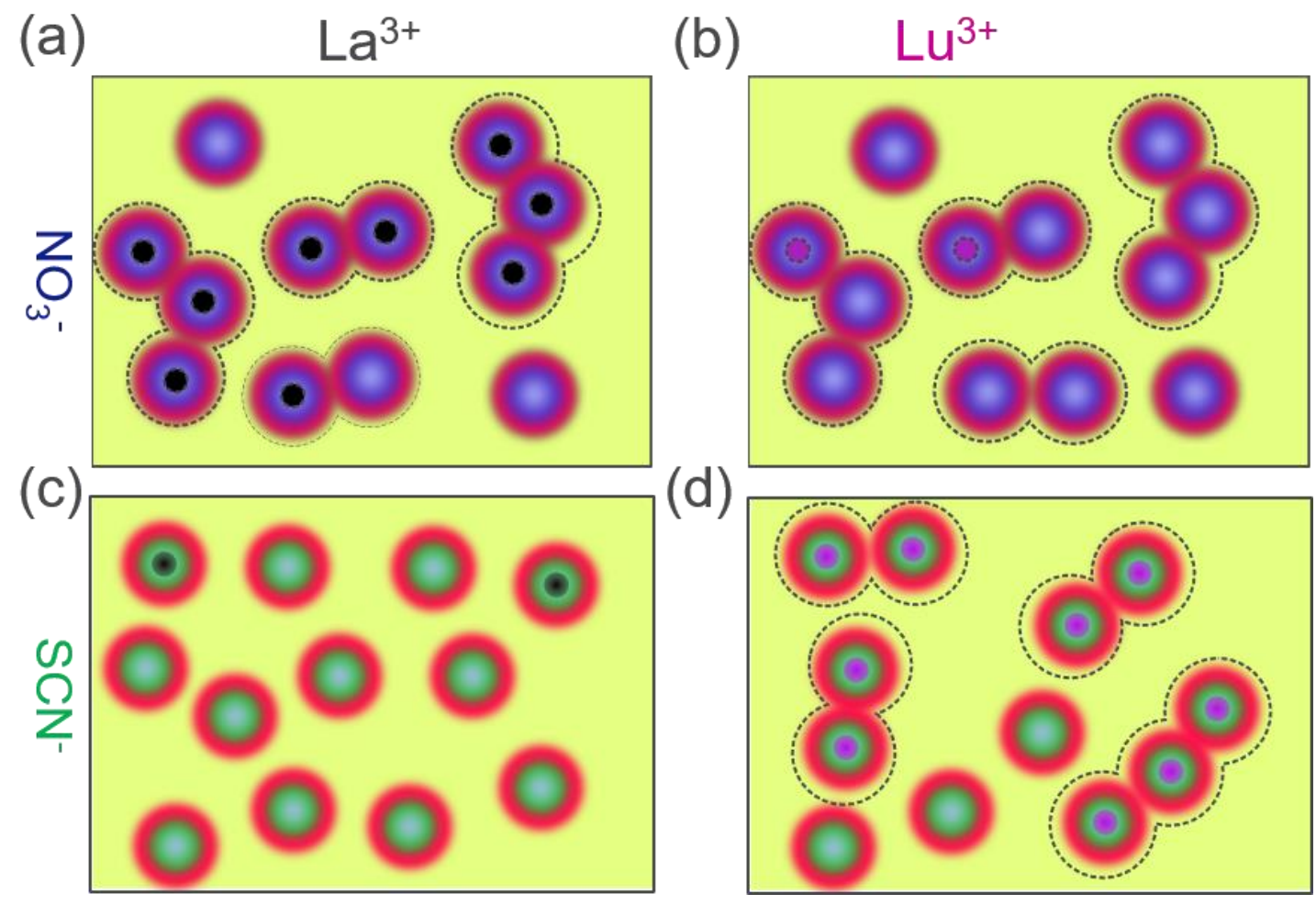

Figure 6. Schematics representing the organic phase structures of (a) $\mathrm{La}^{3+}$ extracted with TOMA$\mathrm{NO}_{3}$, (b) $\mathrm{Lu}^{3+}$ extracted with TOMA-NO 3 , (c) $\mathrm{La}^{3+}$ extracted with TOMA-SCN, and (d) $\mathrm{Lu}^{3+}$ extracted with TOMA-SCN. The clustering behavior in $\mathrm{NO}_{3}^{-}$medium is independent of metal loading, while clustering is observed only with high metal loading in $\mathrm{SCN}^{-}$medium. The black and magenta dots at the center of aggregates represent $\mathrm{La}^{3+}$ and $\mathrm{Lu}^{3+}$ ions, respectively.

The results of our SAXS measurements regarding the SX efficiency of lanthanides and clustering in the organic phase are summarized in Figure 5. In TOMA-NO 3 , clustering is independent of the metal loading (Figure 6a, b). In TOMA-SCN, aggregates can be described as hard spheres in low metal loading (Figure 6c). As the heavier lanthanides are extracted better, the metal loading increases, as well as the clustering (Figure 6d). Therefore, these results provide an important piece of the puzzle in explaining the reverse trends observed in $\mathrm{NO}_{3}{ }^{-}$and $\mathrm{SCN}^{-}$media (Figure 1c, datasets B and D). However, a comprehensive evaluation of the ion transfer, starting from the aqueous 
phase, going through the interface, and ending up in the organic phase, is necessary to explain the details of this complex process. ${ }^{38}$ The role of the liquid-liquid interface in SX process is still an understudied area, in spite of the increasing efforts in recent years. ${ }^{58-60}$

TOMA- $\mathrm{NO}_{3}$ system stands out in all known lanthanide $\mathrm{SX}$ systems, as it preferentially extracts light lanthanides over heavy lanthanides $\left(\mathrm{D}_{\mathrm{La}} / \mathrm{D}_{\mathrm{Lu}} \sim 500\right)$ (Figure 1c, dataset $\left.\mathrm{D}\right)$. Some plausible sources of this preference are differences in aqueous phase speciation, interfacial phenomena, and organic phase speciation and aggregation. Our results with $\mathrm{NO}_{3}{ }^{-}$show that there is no change in aggregation state of the organic phase when extracting different lanthanides. A recent EXAFS study of the organic phase obtained by extraction of lanthanides with $\mathrm{TOMA}-\mathrm{NO}_{3}$ showed that all lanthanides form $\mathrm{Ln}\left(\mathrm{NO}_{3}\right)_{5}{ }^{2-}$ anionic complexes in the organic phase, ${ }^{18}$ eliminating the possibility of inner sphere $\mathrm{NO}_{3}{ }^{-}$coordination leading to any significant differences between lanthanides. Thus, it is likely that the reverse series of lanthanide extraction obtained with nitrates is due to the differences in the aqueous phase, such as enthalpy of dehydration of lanthanides. The (de)hydration of anionic complexes are known to play important roles in the SX process. ${ }^{61-62}$ This interpretation resonates with our recent interfacial work showing that $\mathrm{NO}_{3}{ }^{-}$ions have relatively less impact on interfacial water structure at air/aqueous interface occupied by quaternary amine surfactants. ${ }^{63}$ Any species transferred from the aqueous to the organic phase has to be sufficiently dehydrated. In the nitrate case, since the interfacial water structure (and hence hydration) appears to be unaffected by nitrate, lighter lanthanides which have lower enthalpy of hydration would be better extracted. Interestingly, although the extraction studies show the formation of anionic lanthanide species in the organic phase,,${ }^{18}$ it is not known whether they form in the aqueous phase, or at the interface, or only in the organic phase. Only positively charged lanthanide species have been reported in aqueous $\mathrm{NO}_{3}{ }^{-}$and $\mathrm{SCN}^{-}$media. ${ }^{64-65}$ 
Extraction in TOMA-SCN medium appears to be dominated by electrostatic interactions. This is likely due to strong surface affinity of $\mathrm{SCN}^{-}$which lead to a charge reversal at the interface. So, the extraction follows a mechanism similar to the one observed with acidic extractants. Our recent interfacial studies showed that high concentrations of $\mathrm{SCN}^{-}$dehydrates interfacial layer adjacent to a positively charged monolayer. ${ }^{63}$ This should further diminish the importance of the aqueous phase hydration and cause organic phase structure to dominate the free energy difference. The results showing that clustering plays a role in the case of TOMA-SCN (Figure 6c-d), where attractive interactions between the aggregates increases with increasing lanthanide extraction, supports this explanation. When electrostatic interactions dominate, it is expected that heavier lanthanides, with smaller ionic radius and higher charge density, are extracted better.

The anion-dependent structural differences in TOMA solutions shows that interactions beyond the first coordination sphere of the metal are dependent on the anionic complex being extracted. Although the exact role of these long-range interactions is yet to be clarified, this work adds to the growing body of evidence showing their significance in SX. ${ }^{29,34,66-68}$ Further investigations of these mesoscale phenomena can help elucidate the different extraction trends from $\mathrm{NO}_{3}{ }^{-}$versus $\mathrm{SCN}^{-}$media, and the role of the co-extracted anions in general.

We have investigated an overlooked example of specific ion effects in SX and metal-amphiphile aggregation. Our SAXS experiments elucidated the qualitative differences between the clustering behavior of metal-amphiphile aggregates in the presence of $\mathrm{NO}_{3}{ }^{-}$and $\mathrm{SCN}^{-}$. In order to obtain unambiguous results from SAXS data, we developed a new analytical model for data fitting, which can handle absolute scattering intensities and provide high quality fits with reasonable physical parameters under all conditions. We believe that this model can be used in many SX systems for a better physical understanding. Our results show that clustering in $\mathrm{TOMA}-\mathrm{NO}_{3}$ is independent of 
metal loading, in contrast to metal loading dependent clustering in TOMA-SCN. Therefore, the $\mathrm{SX}$ efficiency is driven by the aqueous phase speciation in $\mathrm{NO}_{3}{ }^{-}$system, in contrast to organic phase driven SX efficiency in $\mathrm{SCN}^{-}$systems. These results show that the ion specific effects in complex fluids require a comprehensive understanding which goes beyond Hofmeister series or similar rankings of ions. Considering that the enthalpic and entropic contributions from various sources are very close to each other in these complex processes, ion specific effects may lead to preference of completely different mechanisms, leading to significant different macroscopic effects.

METHODS

Materials. Methyltrioctylammonium chloride (TOMA-Cl), lanthanide nitrates and chlorides were purchased from Sigma-Aldrich. All the chemicals were used as received without further purification.

Solvent Extraction. TOMA-NO 3 and TOMA-SCN were prepared according to previously described methods. ${ }^{19}$ TOMA concentration was kept at $0.25 \mathrm{M}$ in toluene. Aqueous phase solutions before solvent extraction contained $3 \mathrm{M}$ of $\mathrm{NO}_{3}^{-}$or $\mathrm{SCN}^{-}$, and a desired amount $(2 \mathrm{mM}$ to $0.2 \mathrm{M}$ ) of lanthanide nitrate or chloride respectively. For the background anion, $\mathrm{NaNO}_{3}$ or $\mathrm{NaSCN}$ was used. Equal volumes of the organic phase and the aqueous phases were contacted in a glass vial by vortex mixing for $\sim 1$ minute, followed by radial shaking for one hour at room temperature. The phases were then separated by centrifugation and stored separately in glass vials.

SAXS Experiments. SAXS measurements were conducted at sector 12-ID-C of Advanced Photon Source, Argonne National Laboratory. X-ray energy was $18 \mathrm{keV}(\lambda=0.516 \AA)$. Samples were contained in $2 \mathrm{~mm}$ OD Quartz capillaries. Standard data reduction procedures were used to 
obtain the 1D scattering profiles for the samples. Toluene was used as a standard for absolute intensity calibration.

SAXS data analysis. Background subtracted and calibrated SAXS data was fit to a model of clustering hard-spheres as shown below. Here we model the aggregates as core-shell structures. Due to the high extractant concentration present in our system, the inter-micellar interactions cannot be ignored. We use hard-sphere structure factor $\left(S_{p}(Q)\right)$ based on Percus-Yevick approximation and include a clustering term $\left(P_{c}(Q)\right)$ that arises due to weak attraction between the aggregates. ${ }^{48}$

$$
\begin{gathered}
I_{\text {measured }}=\text { Calibration } * I(Q)+\text { Background } \\
I(Q)=P(Q) S(Q) \\
P(Q)=\frac{\phi_{p}}{V_{s}}\left[\frac{3 V_{c}\left(\rho_{c}-\rho_{s}\right) j\left(Q R_{c}\right)}{Q R_{c}}+\frac{3 V_{s}\left(\rho_{s}-\rho_{\text {solvent }}\right) j\left(Q R_{S}\right)}{Q R_{S}}\right] 2 \\
j(x)=\frac{\sin x-x \cos x}{x^{2}} \\
S(Q)=f P_{c}(Q)+S_{i}(Q)
\end{gathered}
$$

In the above equations $\mathrm{I}(\mathrm{Q})$ is the absolute intensity (scattering cross section) in $\mathrm{cm}^{-1} ; \mathrm{P}(\mathrm{Q})$ is the form factor of scatterers; $\mathrm{S}(\mathrm{Q})$ is the total structure factor; $\phi_{\mathrm{p}}$ is the volume fraction of the scatterers; $R_{c}$ and $R_{s}$ are core and shell radius respectively; $V_{c}$ and $V_{s}$ are volumes of core and shell respectively; $\rho_{\mathrm{c}}, \rho_{\mathrm{s}}$, and $\rho_{\text {solvent }}$ are scattering length densities of core, shell, and solvent respectively. In the structure factor equation, $\mathrm{f}+1$ is the number of aggregates per cluster, $\mathrm{P}_{\mathrm{c}}$ is the form factor of the cluster, and $\mathrm{S}_{\mathrm{p}}$ is the hard-sphere structure factor which is a function of the hardsphere volume fraction $\left(\phi_{H S}\right)$ and size (same as the size of the shell). Form factor of the cluster is obtained using the Guinier-Porod equation. This theoretical curve is fit to the experimental data to 
obtain optimal fit values for $R_{c}, R_{s}, \rho_{c}, \rho_{s}, \phi_{H S}, f, R_{g}$ (radius of gyration of the cluster) and $d$ (Porod exponent of the cluster).

ASSOCIATED CONTENT

\section{Supporting Information.}

The supporting information consists of

The hard sphere fluid structure factor, components of the cluster model fits and tables providing fit parameters from SAXS data, SAXS of TOMA-Cl in toluene, Baxter model fits to the SAXS data. (PDF)

\section{AUTHOR INFORMATION}

\section{Corresponding Author}

*Email: ahmet@anl.gov. Web: www.anl.gov/profile/ahmet-uysal

\section{Author Contributions}

The manuscript was written through contributions of all authors. All authors have given approval to the final version of the manuscript.

\section{Notes}

Authors declare no competing financial interest.

\section{ACKNOWLEDGEMENTS}

We thank Soenke Seifert for his help in conducting SAXS experiments, and Byeongdu Lee for his suggestions in analyzing the SAXS data. This work is supported by the U.S. Department of Energy, Office of Basic Energy Science, Division of Chemical Sciences, Geosciences, and 
Biosciences, under contract DE-AC02-06CH11357. Use of the Advanced Photon Source, an Office of Science User Facility operated for the U.S. Department of Energy (DOE) Office of Science by Argonne National Laboratory, was supported by the U.S. DOE under Contract No. DEAC02-06CH11357.

\section{REFERENCES}

1. Alonso, E.; Sherman, A. M.; Wallington, T. J.; Everson, M. P.; Field, F. R.; Roth, R.;

Kirchain, R. E., Evaluating Rare Earth Element Availability: A Case with Revolutionary Demand from Clean Technologies. Environ. Sci. Technol. 2012, 46, 3406-3414.

2. Charalampides, G.; Vatalis, K. I.; Apostoplos, B.; Ploutarch-Nikolas, B., Rare Earth Elements: Industrial Applications and Economic Dependency of Europe. Procedia Economics and Finance 2015, 24, 126-135.

3. Navarro, J.; Zhao, F., Life-Cycle Assessment of the Production of Rare-Earth Elements for Energy Applications: A Review. Front. Energy Res. 2014, 2.

4. Balaram, V., Rare earth elements: A review of applications, occurrence, exploration, analysis, recycling, and environmental impact. Geosci. Front. 2019, 10, 1285-1303.

5. Gupta, C. K.; Krishnamurthy, N., Extractive Metallurgy Of Rare-Earths. Int. Mater. Rev. 1992, 37, 197-248.

6. Xie, F.; Zhang, T. A.; Dreisinger, D.; Doyle, F., A critical review on solvent extraction of rare earths from aqueous solutions. Miner. Eng. 2014, 56, 10-28.

7. Jha, M. K.; Kumari, A.; Panda, R.; Rajesh Kumar, J.; Yoo, K.; Lee, J. Y., Review on hydrometallurgical recovery of rare earth metals. Hydrometallurgy 2016, 165, 2-26. 
8. National Academies of Sciences, E.; Medicine, A Research Agenda for Transforming Separation Science. The National Academies Press: Washington, DC, 2019.

9. Peppard, D. F.; Mason, G. W.; Maier, J. L.; Driscoll, W. J., Fractional extraction of the lanthanides as their di-alkyl orthophosphates. J. Inorg. Nucl. Chem. 1957, 4, 334-343.

10. Kolař́ík, Z.; Pánková, H., Acidic organophosphorus extractants-I Extraction of lanthanides by means of dialkyl phosphoric acids—effect of structure and size of alkyl group. $J$. Inorg. Nucl. Chem. 1966, 28, 2325-2333.

11. Sato, T., Liquid Liquid Extraction of Rare-Earth Elements from Aqueous Acid-Solutions by Acid Organo-Phosphorus Compounds. Hydrometallurgy 1989, 22, 121-140.

12. Wilson, R. E.; Carter, T. J.; Autillo, M.; Stegman, S., Thiocyanate complexes of the lanthanides, Am and Cm. Chem. Commun. 2020, 56, 2622-2625.

13. Preston, J. S., and Du Preez, A. C., Solvent-extraction processes for the separation of the rare-earth metals. In: T. Sekine (Editor), Solvent Extraction 1990, 883.

14. Brigham, D. M.; Ivanov, A. S.; Moyer, B. A.; Delmau, L. H.; Bryantsev, V. S.; Ellis, R. J., Trefoil-Shaped Outer-Sphere Ion Clusters Mediate Lanthanide(III) Ion Transport with Diglycolamide Ligands. J. Am. Chem. Soc. 2017, 139, 17350-17358.

15. Baldwin, A. G.; Ivanov, A. S.; Williams, N. J.; Ellis, R. J.; Moyer, B. A.; Bryantsev, V. S.; Shafer, J. C., Outer-Sphere Water Clusters Tune the Lanthanide Selectivity of Diglycolamides. ACS Cent. Sci. 2018, 4, 739-747.

16. Gaudernack, B.; Hannestad, G.; Hundere, I. Process for separation of yttrium from the lanthanides. 1974, U.S. Patent No. 3,821,352. 
17. Larsson, K.; Binnemans, K., Separation of rare earths by split-anion extraction. Hydrometallurgy 2015, 156, 206-214.

18. Onghena, B.; Papagni, E.; Souza, E. R.; Banerjee, D.; Binnemans, K.; Vander Hoogerstraete, T., Speciation of lanthanide ions in the organic phase after extraction from nitrate media by basic extractants. Rsc. Adv. 2018, 8, 32044-32054.

19. Knight, A. W.; Chiarizia, R.; Soderholm, L., Extraction Selectivity of a Quaternary Alkylammonium Salt for Trivalent Actinides over Trivalent Lanthanides: Does Extractant Aggregation Play a Role? Solvent Extr. Ion Exc. 2017, 35, 266-279.

20. Sun, P.; Huang, K.; Liu, H., The nature of salt effect in enhancing the extraction of rare earths by non-functional ionic liquids: Synergism of salt anion complexation and Hofmeister bias. J. Colloid Interf. Sci. 2019, 539, 214-222.

21. Lommelen, R.; Vander Hoogerstraete, T.; Onghena, B.; Billard, I.; Binnemans, K., Model for Metal Extraction from Chloride Media with Basic Extractants: A Coordination Chemistry Approach. Inorg. Chem. 2019, 58, 12289-12301.

22. Zhang, S.; Zhao, Y., Facile Preparation of Organic Nanoparticles by Interfacial CrossLinking of Reverse Micelles and Template Synthesis of Subnanometer Au-Pt Nanoparticles. ACS Nano 2011, 5, 2637-2646.

23. Graham, T. R.; Renslow, R.; Goyind, N.; Saunders, S. R., Precursor Ion-Ion Aggregation in the Brust-Schiffrin Synthesis of Alkanethiol Nanoparticles. J. Phys. Chem. C 2016, 120, 1983719847. 
24. Erlinger, C.; Gazeau, D.; Zemb, T.; Madic, C.; Lefrancois, L.; Hebrant, M.; Tondre, C., Effect of nitric acid extraction on phase behavior, microstructure and interactions between primary aggregates in the system dimethyldibutyltetradecylmalonamide (DMDBTDMA) n-dodecane water: A phase analysis and small angle X-ray scattering (SAXS) characterisation study. Solvent Extr. Ion Exc. 1998, 16, 707-738.

25. Erlinger, C.; Belloni, L.; Zemb, T.; Madic, C., Attractive interactions between reverse aggregates and phase separation in concentrated malonamide extractant solutions. Langmuir 1999, $15,2290-2300$.

26. Chiarizia, R.; Nash, K. L.; Jensen, M. P.; Thiyagarajan, P.; Littrell, K. C., Application of the Baxter model for hard spheres with surface adhesion to SANS data for the U(VI)-HNO3, TBPn-dodecane system. Langmuir 2003, 19, 9592-9599.

27. Nave, S.; Modolo, G.; Madic, C.; Testard, F., Aggregation properties of N,N,N',N'tetraoctyl-3-oxapentanediamide (TODGA) in n-dodecane. Solvent Extr. Ion Exc. 2004, 22, 527551.

28. Antonio, M. R.; Chiarizia, R.; Gannaz, B.; Berthon, L.; Zorz, N.; Hill, C.; Cote, G., Aggregation in solvent extraction systems containing a malonamide, a dialkylphosphoric acid and their mixtures. Sep. Sci. Technol 2008, 43, 2572-2605.

29. Ellis, R. J.; Audras, M.; Antonio, M. R., Mesoscopic Aspects of Phase Transitions in a Solvent Extraction System. Langmuir 2012, 28, 15498-15504. 
30. Anderson, T. L.; Braatz, A.; Ellis, R. J.; Antonio, M. R.; Nilsson, M., Synergistic Extraction of Dysprosium and Aggregate Formation in Solvent Extraction Systems Combining TBP and HDBP. Solvent Extr. Ion Exc. 2013, 31, 617-633.

31. Ellis, R. J.; Anderson, T. L.; Antonio, M. R.; Braatz, A.; Nilsson, M., A SAXS Study of Aggregation in the Synergistic TBP-HDBP Solvent Extraction System. J Phys. Chem. B 2013, $117,5916-5924$.

32. Ellis, R. J.; Meridiano, Y.; Chiarizia, R.; Berthon, L.; Muller, J.; Couston, L.; Antonio, M. R., Periodic Behavior of Lanthanide Coordination within Reverse Micelles. Chem. Eur. J. 2013, $19,2663-2675$.

33. Ellis, R. J., Critical Exponents for Solvent Extraction Resolved Using SAXS. J. Phys. Chem. B 2014, 118, 315-322.

34. Ellis, R. J.; Meridiano, Y.; Muller, J.; Berthon, L.; Guilbaud, P.; Zorz, N.; Antonio, M. R.; Demars, T.; Zemb, T., Complexation-induced supramolecular assembly drives metal-ion extraction. Chem. Eur. J. 2014, 20, 12796-807.

35. Bera, M. K.; Ellis, R. J.; Burton-Pye, B. P.; Antonio, M. R., Structural aspects of heteropolyacid microemulsions. Phys. Chem. Chem. Phys. 2014, 16, 22566-74.

36. Ferru, G.; Gomes Rodrigues, D.; Berthon, L.; Diat, O.; Bauduin, P.; Guilbaud, P., Elucidation of the Structure of Organic Solutions in Solvent Extraction by Combining Molecular Dynamics and X-ray Scattering. Angew. Chem., Int. Ed. 2014, 53, 5346-5350.

37. Ferru, G.; Reinhart, B.; Bera, M. K.; Olvera de la Cruz, M.; Qiao, B.; Ellis, R. J., The Lanthanide Contraction beyond Coordination Chemistry. Chem. Eur. J. 2016, 22, 6899-6904. 
38. Poirot, R.; Le Goff, X.; Diat, O.; Bourgeois, D.; Meyer, D., Metal Recognition Driven by Weak Interactions: A Case Study in Solvent Extraction. ChemPhysChem 2016, 17, 2112-2117.

39. Antonio, M. R.; Ellis, R. J.; Estes, S. L.; Bera, M. K., Structural insights into the multinuclear speciation of tetravalent cerium in the tri-n-butyl phosphate-n-dodecane solvent extraction system. Phys. Chem. Chem. Phys. 2017, 19, 21304-21316.

40. Knight, A. W.; Qiao, B. F.; Chiarizia, R.; Ferru, G.; Forbes, T.; Ellis, R. J.; Soderholm, L., Subtle Effects of Aliphatic Alcohol Structure on Water Extraction and Solute Aggregation in Biphasic Water/n-Dodecane. Langmuir 2017, 33, 3776-3786.

41. Ellis, R. J., Acid-switched Eu(III) coordination inside reverse aggregates: Insights into a synergistic liquid-liquid extraction system. Inorganica Chim. Acta 2017, 460, 159-164.

42. Motokawa, R.; Kobayashi, T.; Endo, H.; Mu, J.; Williams, C. D.; Masters, A. J.; Antonio, M. R.; Heller, W. T.; Nagao, M., A Telescoping View of Solute Architectures in a Complex Fluid System. ACS Cent. Sci. 2019, 5, 85-96.

43. Baldwin, A. G.; Servis, M. J.; Yang, Y.; Bridges, N. J.; Wu, D. T.; Shafer, J. C., The structure of tributyl phosphate solutions: Nitric acid, uranium (VI), and zirconium (IV). J. Mol. Liq. 2017, 246, 225-235.

44. Mu, J.; Motokawa, R.; Akutsu, K.; Nishitsuji, S.; Masters, A. J., A Novel Microemulsion Phase Transition: Toward the Elucidation of Third-Phase Formation in Spent Nuclear Fuel Reprocessing. J. Phys. Chem. B 2018, 122, 1439-1452.

45. Ellis, R. I.; Antonio, M. R., Coordination Structures and Supramolecular Architectures in a Cerium(III)-Malonamide Solvent Extraction System. Langmuir 2012, 28, 5987-5998. 
46. Qiao, B.; Demars, T.; de la Cruz, M. O.; Ellis, R. J., How Hydrogen Bonds Affect the Growth of Reverse Micelles around Coordinating Metal Ions. J Phys. Chem. Lett. 2014, 5, 14401444.

47. Chiarizia, R.; Jensen, M. P.; Borkowski, M.; Thiyagarajan, P.; Littrell, K. C., Interpretation of Third Phase Formation in the Th(IV)-HNO3, TBP-n-Octane System with Baxter's "Sticky Spheres" Model. Solvent Extr. Ion Exc. 2004, 22, 325-351.

48. Li, X. D.; Charaya, H.; Bernard, G. M.; Elliott, J. A. W.; Michaelis, V. K.; Lee, B.; Chung, H. J., Low-Temperature Ionic Conductivity Enhanced by Disrupted Ice Formation in Polyampholyte Hydrogels. Macromolecules 2018, 51, 2723-2731.

49. Sztucki, M.; Narayanan, T.; Belina, G.; Moussaïd, A.; Pignon, F.; Hoekstra, H., Kinetic arrest and glass-glass transition in short-ranged attractive colloids. Phys. Rev. E 2006, 74, 051504.

50. Zemb, T.; Bauer, C.; Bauduin, P.; Belloni, L.; Déjugnat, C.; Diat, O.; Dubois, V.; Dufrêche, J.-F.; Dourdain, S.; Duvail, M.; Larpent, C.; Testard, F.; Pellet-Rostaing, S., Recycling metals by controlled transfer of ionic species between complex fluids: en route to "ienaics". Colloid Polym. Sci. 2015, 293, 1-22.

51. Kotlarchyk, M.; Chen, S. H., Analysis of small angle neutron scattering spectra from polydisperse interacting colloids. J. Chem. Phys. 1983, 79, 2461-2469.

52. Frenkel, D.; Vos, R. J.; de Kruif, C. G.; Vrij, A., Structure factors of polydisperse systems of hard spheres: A comparison of Monte Carlo simulations and Percus-Yevick theory. J. Chem. Phys. 1986, 84, 4625-4630. 
53. Hopkins Hatzopoulos, M.; Eastoe, J.; Dowding, P. J.; Grillo, I., Cylinder to sphere transition in reverse microemulsions: The effect of hydrotropes. J. Colloid Interf. Sci. 2013, 392, 304-310.

54. Sharma, S.; Ganguli, A. K., Spherical-to-Cylindrical Transformation of Reverse Micelles and Their Templating Effect on the Growth of Nanostructures. J. Phys. Chem. B 2014, 118, $4122-$ 4131.

55. Jensen, G. V.; Lund, R.; Gummel, J.; Narayanan, T.; Pedersen, J. S., Monitoring the Transition from Spherical to Polymer-like Surfactant Micelles Using Small-Angle X-Ray Scattering. Angew. Chem., Int. Ed. 2014, 53, 11524-11528.

56. Chiarizia, R.; Jensen, M. P.; Borkowski, M.; Ferraro, J. R.; Thiyagarajan, P.; Littrell, K. C., Third Phase Formation Revisited: The U(VI), HNO3-TBP, n-Dodecane System. Solvent Extr. Ion Exc. 2003, 21, 1-27.

57. Ruckenstein, E.; Nagarajan, R., Aggregation of amphiphiles in nonaqueous media. J. Phys. Chem. 1980, 84, 1349-1358.

58. Scoppola, E.; Watkins, E. B.; Campbell, R. A.; Konovalov, O.; Girard, L.; Dufrêche, J.-F.; Ferru, G.; Fragneto, G.; Diat, O., Solvent Extraction: Structure of the Liquid-Liquid Interface Containing a Diamide Ligand. Angew. Chem., Int. Ed. 2016, 55, 9326-9330.

59. Liang, Z.; Bu, W.; Schweighofer, K. J.; Walwark, D. J.; Harvey, J. S.; Hanlon, G. R.; Amoanu, D.; Erol, C.; Benjamin, I.; Schlossman, M. L., Nanoscale view of assisted ion transport across the liquid-liquid interface. Proc. Natl. Acad. Sci. US A 2019, 116, 18227-18232. 
60. Chowdhury, A. U. L., Lu; Doughty, Benjamin Hydrogen-Bond Driven Chemical Separations: Elucidating the Inter-facial Steps of Self-Assembly in Solvent Extraction. ChemRxiv. Preprint. 2020, DOI:10.26434/chemrxiv.11926860.v1

61. Uysal, A.; Rock, W.; Qiao, B. F.; Bu, W.; Lin, B. H., Two-Step Adsorption of PtCl62Complexes at a Charged Langmuir Monolayer: Role of Hydration and Ion Correlations. J. Phys. Chem. C 2017, 121, 25377-25383.

62. Rock, W.; Qiao, B. F.; Zhou, T. C.; Clark, A. E.; Uysal, A., Heavy Anionic Complex Creates a Unique Water Structure at a Soft Charged Interface. J. Phys. Chem. C 2018, 122, 2922829236.

63. Lovering, K.; Nayak, S.; Bu, W.; Uysal, A., The Role of Specific Ion Effects in Ion Transport: The Case of Nitrate and Thiocyanate. J. Phys. Chem. C 2020, 124, 573-581.

64. Peppard, D. F.; Mason, G. W.; Hucher, I., Stability constants of certain lanthanide(III) and actinide(III) chloride and nitrate complexes. J. Inorg. Nucl. Chem. 1962, 24, 881-888.

65. Khopkar, P. K.; Mathur, J. N., Thiocyanate complexing of some trivalent actinides and lanthanides. J. Inorg. Nucl. Chem. 1974, 36, 3819-3825.

66. Dufreche, J. F.; Zemb, T., Effect of long-range interactions on ion equilibria in liquidliquid extraction. Chem. Phys. Lett. 2015, 622, 45-49.

67. Spadina, M.; Bohinc, K.; Zemb, T.; Dufreche, J. F., Synergistic Solvent Extraction Is Driven by Entropy. ACS Nano 2019, 13, 13745-13758.

68. Spadina, M.; Bohinc, K.; Zemb, T.; Dufreche, J. F., Multicomponent Model for the Prediction of Nuclear Waste/Rare-Earth Extraction Processes. Langmuir 2018, 34, 10434-10447. 
TABLE OF CONTENTS FIGURE

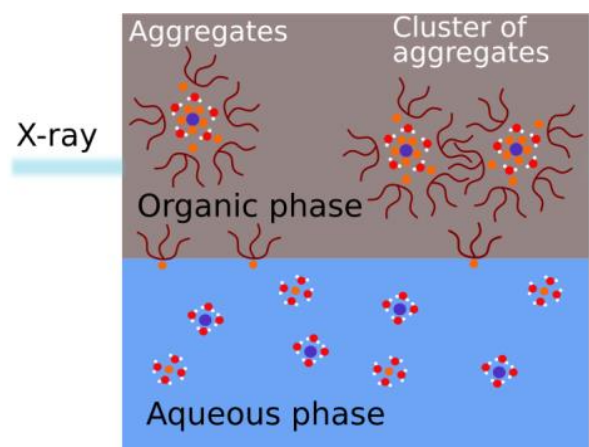

SAXS 